\title{
Ultrasound comparison of the effects of prehabilitation exercises and the scapular assistance test on the acromiohumeral distance
}

\author{
M M Gous, $\mathrm{MTech}^{1}$, B van Dyk, $\mathrm{MTech}^{2}$, E J Bruwer, $\mathrm{PhD}^{3}$ \\ ${ }^{1}$ Department of Medical Imaging and Radiation Sciences, Faculty of Health \\ Sciences, University of Johannesburg, Johannesburg, South Africa \\ ${ }^{2}$ Department of Medical Imaging and Radiation Sciences, Faculty of Health \\ Sciences, University of Johannesburg, Johannesburg, South Africa \\ ${ }^{3}$ Department of Physical Activity, Sport and Recreation (PHASRec), North \\ West University, Potchefstroom, South Africa
}

Corresponding author: MM Gous (magdeleen.gous@gmail.com)

Background: Prolonged participation in overhead sports creates shoulder muscle imbalances which eventually alter the efficacy of the shoulder stabiliser muscles and heighten injury risk, such as subacromial impingement syndrome.

Objectives: The aim of this study was to determine if ultrasound is effective to measure the acromiohumeral distance (AHD) to compare the effect of the scapular assistance test (SAT) on the AHD with a prehabilitative exercise intervention programme in asymptomatic cricket players.

Methods: Baseline testing on cricket players from the NorthWest University cricket squad $(\mathrm{N}=34)$ included AHD measurements performed by a sonographer at $0^{\circ}, 30^{\circ}$ and $60^{\circ}$ humeral abduction angles respectively, with and without the SAT application. Players were then randomly assigned to an intervention and control group. The control group continued with their normal in-season programme, whereas the intervention group also performed shoulder stability exercises for six weeks.

Results: The exercise intervention had a similar effect as the SAT on the AHD at $0^{\circ}$ and $30^{\circ}$ humeral abduction angles in the intervention group. The AHD measurements in the exercise intervention group indicated widening at all abduction angles after the six-week intervention period, whereas the AHD measurements in the control group were equal or smaller than baseline measurements without the SAT at $30^{\circ}$ and $60^{\circ}$ respectively.

Conclusion: Exercise intervention has a similar effect on the AHD of asymptomatic cricket players compared to the SAT especially in $0^{\circ}$ and $30^{\circ}$ of humeral abduction. Ultrasound can therefore be utilised to assist in identifying the risk of developing subacromial impingement syndrome (SIS) in asymptomatic overhead athletes by measuring the AHD at different angles of humeral abduction, without and with the SAT application.

Keywords: Acromiohumeral distance, subacromial impingement syndrome, overhead athletes

S Afr J Sports Med 2017;29:1-6. DOI: 10.17159/2078-516X/2017/v29i0a1396

Subacromial impingement syndrome (SIS) is commonly encountered in overhead athletes when one arm is used in an overhead position. Although a complete throwing motion only lasts a few seconds, repetitive overhead motion creates significant stress on the shoulder, resulting in muscle imbalances which heighten the risk of developing SIS.[1] Prolonged muscle imbalances lead to a narrowing of the acromiohumeral distance (AHD) and painful compression of the soft tissue structures which pass through the subacromial space (SAS) during dynamic humeral abduction. ${ }^{2,3[}$ A late diagnosis of SIS may lead to decreased sport performance while valuable training and competition time is lost due to long periods of rehabilitation. ${ }^{4}$

The scapular assistance test (SAT) is used to identify abnormal scapular motion before late stage winging is present. The examiner manually corrects the dyskinesis and stabilises the scapula on the bony thorax by rotating the scapula upward and outwards, while simultaneously pushing the scapula to increase the posterior tilt during humeral elevation. ${ }^{5,6]}$ The SAT manoeuvre relieves compression on soft tissue structures responsible for SIS by increasing the AHD. The test is therefore positive when the athlete's symptoms are reduced with the abduction of the humerus while the SAT is applied. ${ }^{[5,6,7]}$ If pain reduces with the SAT, it may be assumed that the strengthening of the scapular stabilisers will result in a widening of the AHD and the lessening of the clinical effects of SIS. However, in the sporting world it would be helpful to determine whether the prehabilitation of the shoulder girdle is needed before the onset of impingement symptoms. Determining the AHD, before and after the SAT application, at different angles of humeral abduction in the dominant and non-dominant shoulders of unilateral overhead athletes could provide valuable insight into the type of soft tissue injury and contribute to its mitigation.

There is currently no proven imaging or clinical method to identify the risk of future SIS in asymptomatic overhead athletes. Ultrasound is a non-invasive, comfortable and dynamic examination which provides extensive diagnostic information of the shoulder muscles, as well as the AHD variation, during the abduction of the humerus. ${ }^{8}$ Although ultrasound can accurately measure the AHD, the literature is not clear as to whether it can predict SIS in overhead athletes by measuring the AHD at different humeral abduction angles. ${ }^{8}$

The purpose of this article is to describe the use of ultrasound AHD measurements to compare the effect of SAT on the AHD with that of a prehabilitative exercise intervention in asymptomatic cricket players.

\section{Methods}

\section{Study design and participants}

Male cricket players $(\mathrm{N}=47)$ from the North-West University (NWU) cricket squad were recruited to voluntarily participate in this randomised control trial during the 2013 cricket season. Ethical approval was obtained from the Faculty of Health Sciences Higher Degrees and Research Ethics Committees at the University of Johannesburg (AEC12-01-2013) and the NWU (NWU-00026-12-A1). Players and coaches were thoroughly 
informed regarding the testing procedures and the exercise intervention programmes. Written informed consent was obtained from all participating players prior to baseline ultrasound examinations of both shoulders and participation in the testing procedures and exercise intervention programmes.

Only players of $\geq 17$ and $\leq 25$ years, who did not suffer from any current orthopaedic condition or injury or who were not rehabilitating from any orthopaedic injury, were eligible for inclusion in this study. The study population was randomly allocated to an exercise intervention group and a control group by participants drawing numbers from a box after the baseline testing. Thirty-four participants completed the posttest procedures (exercise intervention group, $n=16$ and control group, $\mathrm{n}=18$ ).

\section{Ultrasound measurements}

Both shoulders of prospective participants were initially examined with ultrasound to rule out any pre-existing pathology. A baseline ultrasound examination was then performed on all participants who met the study criteria. A Japanese manufactured Hitachi Aloka F75 ultrasound unit, equipped with a $7 \mathrm{MHz}-14 \mathrm{MHz}$ linear broadband transducer, was used for this purpose. The AHD of both shoulders was measured at $0^{\circ}, 30^{\circ}$ and $60^{\circ}$ humeral abduction angles in the scapular plane, with and without application of the SAT.

The transducer was positioned in a sagittal plane along the long axis of the supraspinatus tendon and humerus (Fig. 1a). The AHD on the frozen image can then be defined as the shortest linear distance between the antero-inferior tip of the acromion and the greater tubercle of the humeral head (Fig. 1b). ${ }^{[10]}$

The degrees of humeral abduction were measured with a goniometer, placed on the posterior aspect of the shoulder along the long axis of the humerus (Fig. 2a). [3,6,9] AHD measurements of more than $60^{\circ}$ humeral abduction are not possible due to constraints in the imaging technique. ${ }^{[9]}$ The SAT was then applied by a qualified biokineticist who manually rotated the scapula in an upward rotation and posterior tilt during humeral abduction to manually stabilise the scapula on the bony thorax. ${ }^{5,6]}$ The AHD was remeasured at the same $0^{\circ}, 30^{\circ}$ and $60^{\circ}$ humeral abduction angles (Fig. 2ab).

To ensure internal validity, an independent radiologist audited all the ultrasound images to verify that the correct technique had been used consistently in obtaining the required images and in measuring the AHD.

\section{Exercise intervention}

While continuing with the usual in-season cricket training, the intervention group was also subjected to a six-week exercise intervention programme, twice a week for forty minutes at a time, under the supervision of a qualified biokineticist. The exercise intervention programme focused on releasing the pectoralis minor, pectoralis major, latissimus dorsi and posterior capsule, as well as strengthening the scapular and core stabiliser muscles and humeral lateral rotators. Emphasis was placed on maintaining postural control during the execution of all exercises. A register was kept to log each session the athlete attended and only participants who complied with attending two sessions per week throughout the six week period qualified for follow-up testing.

Conversely, the control group carried on with the usual inseason cricket training and exercises under the supervision of the cricket coach, without the additional biokinetic intervention.

\section{Follow-up AHD measurement}

On completion of the prehabilitative exercise programme, the AHD of both shoulders was re-measured as previously described, albeit without the SAT application. These measurements served as comparative information of the increase in AHD measurement achieved both with the SAT application, as well as with the prehabilitation exercises. A comparison of the follow-up AHD measurements without the SAT to the initial AHD measurements with the SAT, was expected to support or refute the hypothesis that the strengthening of the scapula stabilisers act in the same manner as the SAT application.

\section{Statistical analysis}

The statistical analyses were performed using the IBM SPSS v24 programme (IBM Corp. Released 2016. IBM SPSS Statistics for Windows, Version 24.0. Armonk, NY: IBM Corp). Based on the results of the Shapiro-Wilk test and Quantile-Quantile plots, these authors concluded that the sample was normally distributed and therefore parametric statistical methods were used. Independent T-tests were performed to determine if the exercise intervention and control groups differed in basic participant characteristics. Repeated measures ANOVA tests with between-subjects effects (exercise intervention and control groups) and within-subjects effects (dominant vs nondominant shoulders and humeral abduction angles) were performed to indicate the difference in AHD at $0^{\circ}, 30^{\circ}$ and $60^{\circ}$ of humeral abduction for both the dominant and non-dominant shoulders without the SAT at baseline. Lastly, repeated measures ANOVA tests were also performed with betweensubjects effects (intervention and control groups) and withinsubjects effects (AHD at $0^{\circ}, 30^{\circ}$ and $60^{\circ}$ humeral abduction angles at baseline with and without the SAT, as well as the postintervention period without the SAT) to indicate whether the effect of the SAT and the exercise intervention differed.

\section{Results}

The biographical and anthropometrical characteristics of the sample are summarised in Table 1. The groups presented with similar characteristics making them suitable for comparison (Table 1).

In Fig. 3, the change in the baseline AHD measurement, without the SAT at $0^{\circ}, 30^{\circ}$ and $60^{\circ}$ humeral abduction angles, is indicated for both the dominant and non-dominant shoulders of the intervention and control groups. There were no significant three-way or two-way interactions. A significant main effect between the humeral abduction angles $\left(F_{2,64}=43.86\right.$, $\mathrm{p}<0.001$ ) was observed, indicating that the AHD varies irrespective of dominance or group. Multiple comparisons with 

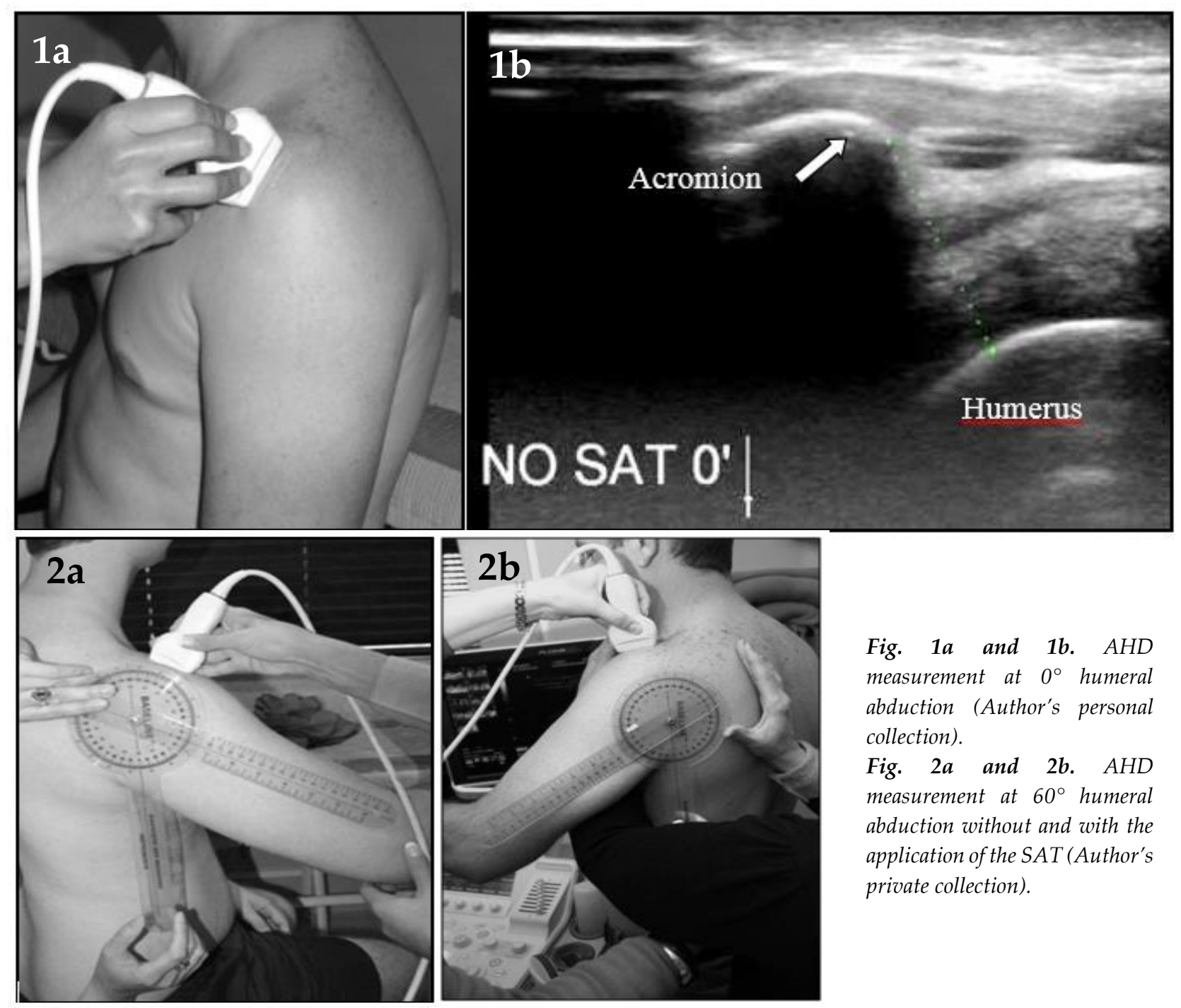

Fig. $1 a$ and 1b. AHD measurement at $0^{\circ}$ humeral abduction (Author's personal collection).

Fig. $2 a$ and $2 b$. AHD measurement at $60^{\circ}$ humeral abduction without and with the application of the SAT (Author's private collection).

a Bonferroni correction was used to indicate that the mean AHD of both groups significantly narrowed during humeral abduction from $0^{\circ}$ to $30^{\circ}(\mathrm{p}=0.002), 0^{\circ}$ to $60^{\circ}(\mathrm{p}<0.001)$ and from $30^{\circ}$ to $60^{\circ}(p<0.001)$. When measured separately, the AHD of both shoulders showed a similar narrowing trend during $0^{\circ}$ to $30^{\circ}$ and $30^{\circ}$ to $60^{\circ}$ humeral abduction.

In Figure 4 the baseline AHD measurement of the dominant shoulder (throwing arm) without SAT of the exercise intervention and control groups was compared to the baseline AHD with SAT and after the intervention without SAT at $0^{\circ}$, $30^{\circ}$ and $60^{\circ}$ humeral abduction angles.

There were no significant three-way interactions observed between $0^{\circ}, 30^{\circ}$ and $60^{\circ}$ humeral abduction angles and the application of the SAT at baseline of the exercise intervention or control groups. Significant two-way interactions were observed between the $0^{\circ}, 30^{\circ}$ and $60^{\circ}$ humeral abduction angles and application of SAT at baseline of the exercise intervention $\left(\mathrm{F}_{2,30}=3.810, \mathrm{p}=0.034\right)$ and control $\left(\mathrm{F}_{2.34}=5.100\right.$, $\mathrm{p}=0.012$ ) groups, indicating that the AHD with the SAT increases significantly during $0^{\circ}, 30^{\circ}$ and $60^{\circ}$ humeral abduction angles compared to the AHD without the SAT at similar abduction angles. Multiple comparisons with a Bonferroni correction was used to indicate that the mean AHD of the intervention group widened with the SAT at humeral abduction angles of $0^{\circ}(\mathrm{p}=0.020), 30^{\circ}(\mathrm{p}=0.031)$ and $60^{\circ}(\mathrm{p}=0.047)$. The mean AHD of the control group widened with the SAT at humeral abduction angles of $0^{\circ}(\mathrm{p}<0.001), 30^{\circ}(\mathrm{p}=0.002)$ and $60^{\circ}$ $(\mathrm{p}<0.001)$. The AHD was of medium practical significance $(\mathrm{d}-$ values $)$ at $0^{\circ}(\mathrm{d}=0.50)$, small to medium practical significance at $30^{\circ}(\mathrm{d}=0.45)$, and of medium to large practical significance at $60^{\circ}(d=0.76)$ abduction in the intervention group. The AHD was of medium practical significance ( $d$-values) at $0^{\circ}(\mathrm{d}=0.50)$, of small to medium practical significance at $30^{\circ}(d=0.43)$, and of large practical significance at $60^{\circ}(d=0.85)$ abduction in the control group.

There were no significant two-way interactions observed between the AHD of the exercise intervention $\left(\mathrm{F}_{2,30}=0.066\right.$, $\mathrm{p}=0.936)$ or control groups $\left(\mathrm{F}_{2,1}=1.292, \mathrm{p}=0.748\right)$ at humeral abduction angles of $0^{\circ}, 30^{\circ}$ and $60^{\circ}$ without the SAT at baseline and the AHD at similar humeral abduction angles without the SAT after the six-week exercise intervention period.

There were no significant three-way interactions observed between the AHD with the SAT at baseline and the AHD without the SAT after the six-week exercise intervention period at humeral abduction angles of $0^{\circ}, 30^{\circ}$ and $60^{\circ}$ of the exercise intervention or control groups. Significant two-way 
Table 1. Comparison of biographical characteristics between the exercise intervention and control groups $(\mathrm{N}=34)$

\begin{tabular}{lccc}
\hline \multicolumn{1}{c}{ Variables } & Intervention (n=16) & Control (n=18) & P-value \\
\hline Age (Years) & $20.7 \pm 1.3$ & $20.9 \pm 1.5$ & 0.826 \\
Height (m) & $1.81 \pm 0.07$ & $1.79 \pm 0.07$ & 0.202 \\
Body mass (kg) & $81.4 \pm 9.4$ & $77.5 \pm 10.3$ & 0.315 \\
BMI (kg.m-2) & $24.7 \pm 1.7$ & $24.0 \pm 2.3$ & 0.786 \\
Number of years participating & & & \\
$<4$ years & $1(6 \%)$ & $1(6 \%)$ & 0.957 \\
$\geq 4$ years & $15(94 \%)$ & $17(94 \%)$ & \\
Participating Level & & & \\
University squad & $12(75 \%)$ & $15(83 \%)$ & \\
Provincial level & $4(25 \%)$ & $3(17 \%)$ & \\
Average Training Hours per week & & & \\
$<6$ hours & $4(25 \%)$ & $7(39 \%)$ & 0.1636 \\
$\geq 6$ hours & $12(75 \%)$ & $11(61 \%)$ & \\
History of Resistance Training & & & \\
$<3$ years & $10(63 \%)$ & $7(39 \%)$ & 0.182 \\
$\geq 3$ years & $6(38 \%)$ & $11(61 \%)$ & \\
\hline
\end{tabular}

Values reported as either mean $\pm s d$ or as number of participants (\% of total participants).

Significance is set at $p<0.05$

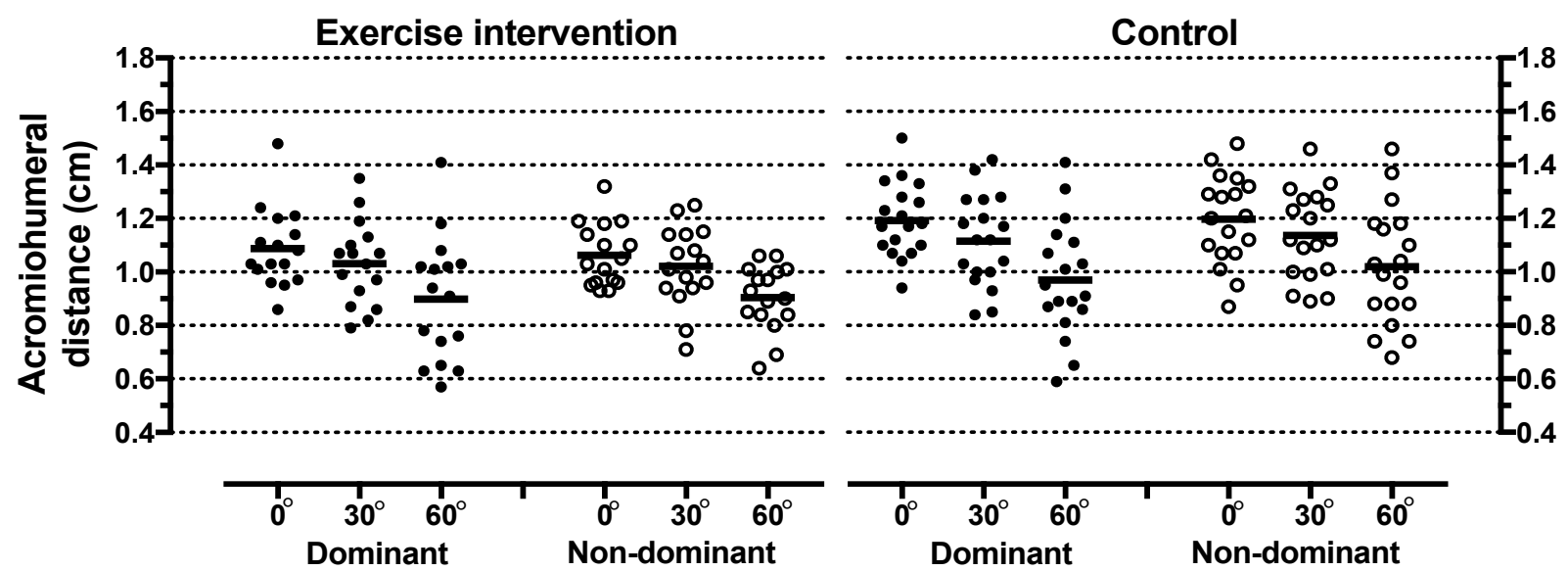

Fig. 3. Comparison of baseline AHD measurements at $0^{\circ}, 30^{\circ}$ and $60^{\circ}$ humeral abduction without the SAT

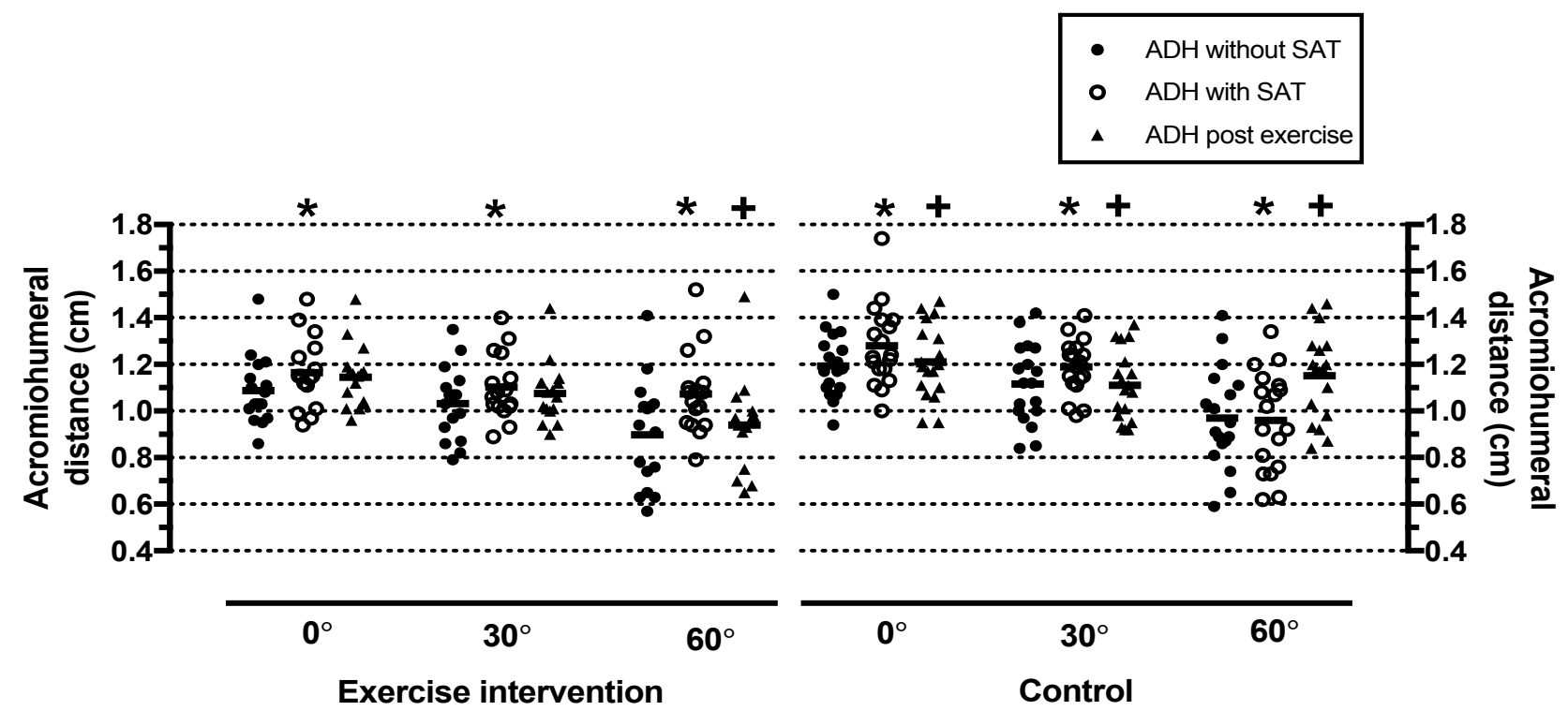

Humeral abduction angles (degrees)

Fig. 4. Comparison of baseline AHD of the exercise intervention and control groups with and without the SAT and after intervention without the $S A T$ at $0^{\circ}, 30^{\circ}$ and $60^{\circ}$ of humeral abduction 
interactions were observed between the AHD with the SAT at baseline and the AHD without the SAT after the six-week intervention period at $0^{\circ}, 30^{\circ}$ and $60^{\circ}$ humeral abduction angles of the exercise intervention $\left(\mathrm{F}_{2,30}=5.979, \mathrm{p}=0.007\right)$ and control $\left(\mathrm{F}_{2,34}=5.909, \mathrm{p}=0.006\right)$ groups.

Multiple comparisons with a Bonferroni correction was used to indicate that there is no significant difference between the AHD with SAT at baseline and the AHD without the SAT after the six-week intervention period in the exercise intervention group at $0^{\circ}(\mathrm{p}=0.538)$ or $30^{\circ}(\mathrm{p}=0.396)$ humeral abduction angles. The AHD of the exercise intervention group does, however, differ statistically at $60^{\circ}(\mathrm{p}=0.001)$ humeral abduction angle. The AHD of the control group at baseline with the SAT does differ statistically from the AHD without the SAT after the six-week study period at $0^{\circ}(\mathrm{p}=0.015), 30^{\circ}(\mathrm{p}=0.010)$ and $60^{\circ}$ $(\mathrm{p}=0.001)$ humeral abduction angles. Note however, no significant difference was observed between the effect of the SAT and the exercise intervention on the AHD at $0^{\circ}$ and $30^{\circ}$ of humeral abduction in the intervention group, thus indicating that the six-week exercise intervention has a similar effect on the AHD at these angles as when the SAT was applied. The AHD of the control group after the intervention period was significantly less than when the SAT was applied, indicating that normal conditioning regimes did not have any positive effects on the AHD.

\section{Discussion}

The study aimed to test the hypothesis that prehabilitative exercises would correct upper body postural adaptations and shoulder muscle imbalances in a similar way as the SAT manually alters the orientation of the scapula to increase the AHD. This was achieved by comparing the effect of the SAT on the AHD to the initial baseline measurements without the $S A T$ at $0^{\circ}, 30^{\circ}$ and $60^{\circ}$ humeral abduction angles and also to the effect of a six-week biokinetics prehabilitation intervention programme on AHD measurements without the SAT.

The baseline AHD measurements of the dominant and nondominant shoulders were almost identical in both groups before the SAT was applied. The assumption that the application of the SAT would increase the AHD in both groups at baseline was proved correct. The greatest effect of the SAT was, however, observed in the dominant shoulder of overhead athletes. Weakness of the serratus anterior and upper or lower trapezius muscles, as well tightness of the posterior capsule, result in anterior tipping of the scapula with subsequent SIS. ${ }^{111,12]}$ The SAT is believed to alter the scapular position through manual upward rotation and posterior tipping to increase the AHD, thus limiting compression of the rotator cuff and subacromial-subdeltoid bursa in the same way that prehabilitation exercises would strengthen the scapular stabiliser muscles and reposition the scapula to prevent SIS. $[2,3,5,13,14]$

When the mean AHD with the SAT was compared to the AHD without the SAT, after completion of the six-week prehabilitation programme, widening of the AHD was evident in the exercise intervention group at $0^{\circ}$ and $30^{\circ}$ humeral abduction angles - most significantly at $30^{\circ}$ of humeral abduction. This implies that the intervention had the same effect on the AHD as the SAT by mitigating muscle imbalances associated with a repeated overhead motion resulting in a smaller chance of developing SIS.

The same general trend was not observed in the control group. With AHD distances smaller than the initial baseline measurements with the SAT, it can be deduced that normal training had no positive effect on the AHD in the control group. On the contrary, it seems as if the reduced AHD in the dominant shoulders at $0^{\circ}, 30^{\circ}$ and $60^{\circ}$ abduction suggests scapular imbalances which may result in SIS over time. A study by Silva et al. ${ }^{[4]}$ similarly reported that asymptomatic tennis players with postural adaptations presented with a smaller AHD than control non-playing participants, as well as tennis players without shoulder dyskinesia which highlights the importance of preventative exercises to limit the condition.

The results of this study thus suggest that AHD measurements of the intervention group without the SAT application after a six-week intervention period are wider than baseline AHD measurements without the SAT at all angles of humeral abduction, and almost similar to AHD measurements with the SAT at baseline. These measurements, therefore, confirm that scapular stabilisation prehabilitation exercises do act in a similar manner as the SAT. As a non-invasive, nonradiating and dynamic examination, ultrasound provides extensive diagnostic information of the shoulder muscles, as well as the variation in the AHD, during abduction of the humerus. ${ }^{[8]}$ Accurate measurements over $60^{\circ}$ can, unfortunately, not be provided due to constraints in the imaging technique. [8,9,10,15] Furthermore, musculoskeletal ultrasound examinations are operator-dependent which may render less accurate results when performed by a less experienced operator. Nevertheless, this study indicated that a different interdisciplinary approach may be useful in the conservative treatment of SIS. Orthopaedic surgeons and sports physicians may consider referring patients with secondary shoulder impingement symptoms, for ultrasound imaging which includes a measurement of the AHD when the SAT is applied. A conservative exercise rehabilitation programme, prescribed by a biokineticst, could then be followed for a predetermined period of time (six weeks in this study) in the hope of mitigating surgical intervention.

\section{Conclusion}

This research aimed to describe the use of ultrasound AHD measurements to compare the effect of the SAT on the AHD with that of a prehabilitative exercise intervention in asymptomatic cricket players. The results of this study indicate that exercise intervention has a similar effect on the AHD of asymptomatic cricket players as the SAT especially in $0^{\circ}$ of humeral abduction. Ultrasound can, therefore, be utilised to assist in identifying the risk of developing SIS in asymptomatic overhead athletes, by measuring the AHD at different angles of humeral abduction, without and with the SAT application. With the SAT having a more pronounced effect on the dominant shoulder as compared to the non-dominant shoulder, it can be expected that the athlete may develop SIS in future as a 
result of postural adaptations and scapular muscle imbalances already present.

The findings of this study propose the use of a standardised ultrasound protocol for the evaluation of the shoulder in unilateral overhead athletes. A multidisciplinary team, consisting of biokineticists, sport scientists, coaches and sonographers, are encouraged to consider the use of high frequency sound imaging as an additional screening tool for the timely detection of SIS risk and correction of muscle imbalances before the onset of symptoms. Such a protocol has the ability to pre-empt decreased sport performance and loss of valuable training and competition time at a competitive level due to injury.

Acknowledgements: The authors wish to acknowledge Ms Marike Cockeran, School for Computer, Statistical and Mathematical Sciences, North-West University, for her statistical support.

\section{References}

1. Kirchhoff $\mathrm{C}$, Imhoff AB. Posterosuperior and anterosuperior impingement of the shoulder in overhead athletes - evolving concepts. Int Ortop 2010;34:1049-1058. [doi: 10.1007/s00264010-1038-0]

2. Kibler WB. Current Concepts: The role of the scapula in athletic shoulder function, Am J Sports Med 1998;26:325-337. [DOI: 10.1177/03635465980260022801]

3. Seitz AL, McClure PW, Finucane S, et al. The scapular assistance test results in changes in scapular position and subacromial space but not rotator cuff strength in subacromial impingement, J Orthop Sports Phys Ther 2012;42:400-412. [doi:10.2519/jospt.2012.3579]

4. Silva RT, Hartman LG, de Souza LCF, et al. Clinical and ultrasonographic correlation between scapular dyskinesia and subacromial space measurement among junior elite tennis players, Br J Sports Med 2010;44:407-410. [doi: 10.1136/bjsm.2008.046284]

5. Sauers EL. Clinical evaluation of scapular dysfunction, Athl Ther Today 2006;11:10-14. [https:// doi.org/10.1123/att.11.5.10]
6. Seitz PL, McClure PW, Lynch SS,et al. Effects of scapular dyskinesis and scapular assistance test on subacromial space during static arm elevation, J Shoulder Elbow Surg 2012;21:631-640. [doi: 10.1016/j.jse.2011.01.008]

7. Phadke V, Camargo P, Ludewig P. Scapular and rotator cuff muscle activity during arm elevation: A review of normal function and alterations with shoulder impingement, Rev Bras Fisioter 2009;13:1-9. [DOI: 10.1590/S1413-35552009005000012]

8. Azzoni R, Cabitza P, Parrini M. Sonographic evaluation of subacromial space. Ultrasonics 2004;42:683-687. [DOI: 10.1016/j.ultras.2003.11.015]

9. Desmeules F, Minville L, Riederer B,et al.. Acromio-humeral distance variation measured by ultrasonography and its association with the outcome of rehabilitation for shoulder impingement syndrome. Clin J Sport Med 2004;14:197-205. [https://doi.org/10.1097/00042752-200407000-00002]

10. Kumar P, Bradley M, Swinkels A. Within-day and day-to-day intrarater reliability of ultrasonographic measurements of acromion-greater tuberosity distance in healthy people, Physiother Theory Pract 2010;26:347-351. [doi: 10.3109/09593980903059522]

11. Miller MD, Thompson SR. DeLee \& Drez's Orthopaedic Sports Medicine: Principles and Practice. 3rd ed. Philadelphia: Saunders, 2010:892.

12. Muraki T, Yamamoto N, Zhao KD, et al. Effects of posterior tightness on subacromial contact behavior during shoulder motions, J Shoulder Elbow Surg 2012;21:1160-1167. [doi:10.1016/j.jse.2011.08.042]

13. Rabin A, Irrgang JJ, Fitzgerald GK, et al. The intertester reliability of the Scapular Assistance Test, J Orthop Sports Phys Ther 2006;36:653-660. [DOI:10.2519/jospt.2006.2234]

14. Kibler BW, McMullen J. Scapular dyskinesis and its relation to shoulder pain, J Am Acad Orthop Surg 2003;11:142-151. [https:// doi.org/10.5435/00124635-200303000-00008]

15. Cholewinski JJ, Kusz DJ, Wojciechowski P, et al. Ultrasound measurement of rotator cuff thickness and acromio-humeral distance in the diagnosis of subacromial impingement syndrome of the shoulder, Knee Surg Sports Traumatol Arthrosc 2007;16:408-414. [DOI: 10.1007/s00167-007-0443-4] 